\title{
Acetyl-coenzyme A carboxylase - an attractive enzyme for biotechnology
}

\author{
JAN PODKOWIŃski *, ALEKSANDER TWORAK \\ Laboratory of Plant Molecular Biology, Institute of Bioorganic Chemistry, PAS, Poznań, Poland \\ * Corresponding author: jpodkow@ibch.poznan.pl
}

\begin{abstract}
Acetyl-CoA carboxylase catalyzes the carboxylation of acetyl-CoA to malonyl-CoA. This reaction constitutes the first committed step of fatty acid synthesis in most organisms, while its product also serves as a universal precursor for various other high-value compounds. The important regulatory and rate-limiting role of acetyl-CoA carboxylase makes this enzyme a powerful tool in a variety of biotechnological and medical projects. This review presents the current knowledge on structural and functional features of the enzyme and focuses on its divergent applications. Different metabolic engineering attempts that lead to the production of various compounds, such as fatty acids, polyketides or flavonoids, are presented and their advantages and limitations discussed. The importance of studies on acetyl-CoA carboxylase activity for design and development of new herbicides and antibiotics as well as for medical intervention is also highlighted.
\end{abstract}

Key words: acetyl-coenzyme A carboxylase, fatty acid, metabolic engineering, biotechnology

\author{
Abbreviations \\ Aas - Acyl-acyl carrier protein synthetase \\ ACCase - Acetyl-coenzyme A carboxylase \\ ACP - Acyl carrier protein \\ Acs - Acetyl-coenzyme A synthetase \\ BC - Biotin carboxylase \\ BCCP - Biotin carboxyl carrier protein
}

$\begin{array}{ll}\text { CoA } & \text { - Coenzyme A } \\ \text { CT } & \text { - Carboxyl transferase } \\ \text { FA } & \text { - fatty acids } \\ \text { FAS } & \text { - fatty acid synthesis } \\ \text { FFA } & \text { - Free fatty acid } \\ \text { TAG } & \text { - triacylglycerol }\end{array}$

Acetyl-coenzyme A carboxylase - enzyme architecture, biochemistry and its role in cell physiology

A variety of biotechnological projects targeted to modulate acetyl-coenzyme A carboxylase activity in bacteria, plants and humans have been proposed and experimentally tested. Here, we would like to present a comprehensive review of these strategies together with a survey of the potential of acetyl-CoA carboxylase for biotechnology.

Acetyl-coenzyme A carboxylase (ACC, E.C.6.4.1.2), recognized as an essential enzyme for all Eukaryotes and the majority of Bacteria, is responsible for carboxylation of acetyl-CoA that results in malonyl-coenzyme A formation (Fig. 1). Malonyl-CoA is a major building block for compounds such as fatty acids, polyketides and flavonoids - important components for cell growth, as well as for food, pharmaceuticals and energy production.
The malonyl-CoA synthesis consists of two half-reactions (Nikolau et al., 2003). The first one, adenosine triphosphate-dependent carboxylation of biotin prosthetic group covalently bound to a module named biotin carboxyl carrier protein (BCCP), is catalyzed by ACCase segment of biotin carboxylase activity (BC) (Fig. 2). This reaction is immediately followed by the second half-re action: the transfer of a carboxyl group from carboxylated biotin to acetyl-CoA, resulting in malonyl-CoA formation. Carboxyl transferase (CT) is a module that catalyzes the second reaction and provides the required specificity in recognition of the carboxyl acceptor (acetyl-CoA). The ACCase model usually presents BCCP, a module with covalently attached biotin, as a beam responsible for biotin dislocation between two active centers - one with BT and the other with CT activity. The three 


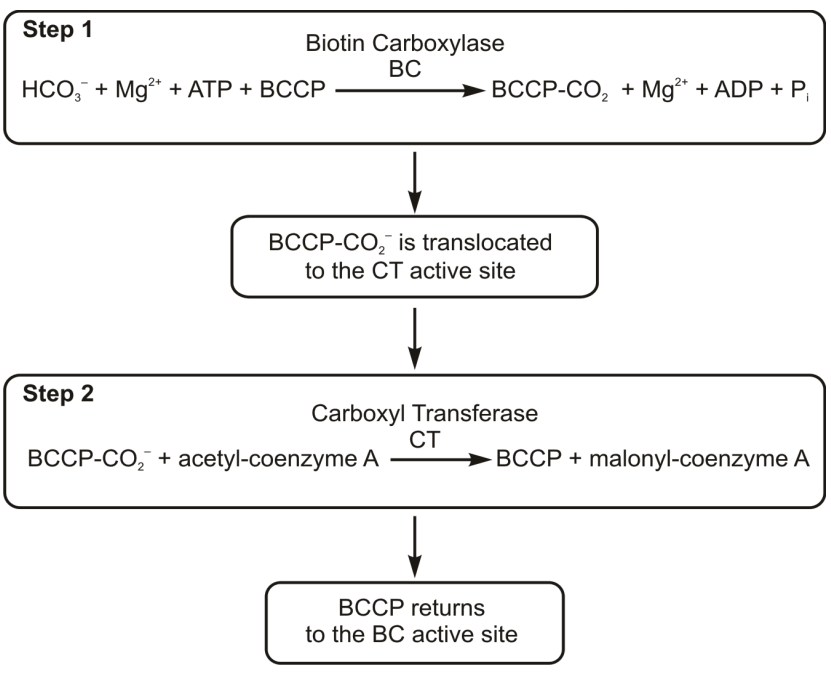

Fig. 1. Acetyl-coenzyme A carboxylation consists of two halfreactions resulting in malonyl-CoA synthesis. The first half reaction is adenosine triphosphate dependent carboxylation of a biotin prosthetic group and second one - transcarboxylation of acetyl-CoA. Bicarbonate ion and acetyl coenzyme A are substrates, ATP and magnesium ions are required for the first half reaction - biotin carboxylation

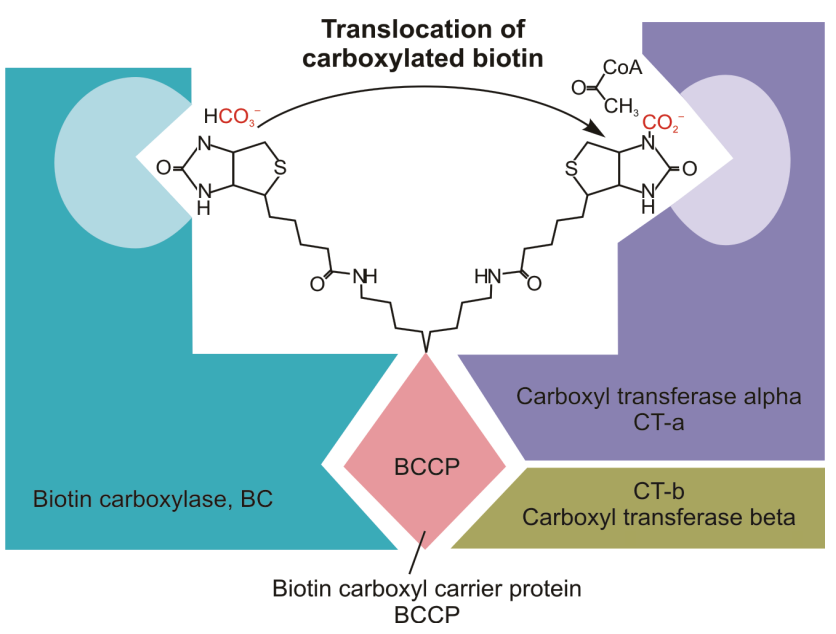

Fig. 2. Schematic model of acetyl-coenzyme A carboxylase composed of four separate subunits - prokaryotic type enzyme. Biotin is responsible for carboxyl group translocation between two active centers - biotin carboxylase and carboxyl transferase

modules are formed either by separate peptides, as in prokaryotic ACCases, or by domains within a single, long peptide as in eukaryotic type ACCase (Fig. 3). The prokaryotic and eukaryotic types of ACCases are evolutionary related and the phylogeny of the modules provides some hints about their cenancestor, a split between Archaea and Bacteria, and arising of Eukaryota (Lombard and Moreira, 2011).
Phylogeny is out of scope of this manuscript, but acetyl-coenzyme A carboxylases from various organisms representing various domains of life are discussed in this review. While, traditionally two abbreviations are in use for acetyl-coenzyme A carboxylase: ACCase for plant enzymes and ACC for human (metazoan) ones, combining them (often in the same paragraph or even sentence) may be confusing. We decide to use consistently one abbreviation and the enzyme name is replaced with "ACCase" when necessary.

Bacteria have a prokaryotic type ACCase with a characteristic separation of carboxyl transferase module into two different peptides: alpha- and beta-CT, coded by two distinct genes. Such ACCase structure is also preserved in plastids owing to their bacterial origin. Plant cells are exceptional among the Eukaryotes that have both types of ACCases: the eukaryotic type residing in cytosol and the prokaryotic one in plastids (Sasaki and Nagano, 2004; Gornicki et al., 1997; Gornicki et al., 1994; Podkowinski et al., 1996). Plastidic ACCase produces malonyl-CoA, the sole source of carbon for de novo fatty acid synthesis (FAS) (Rawsthorne, 2002). Plastidproduced malonyl-CoA is separated from cytosolic pathways as there are no mechanisms for its transport across plastid membranes. Plastid ACCase activity is usually much higher than the cytosolic one due to high demand for fatty acids that are used in plant cells either as components of cell membranes or storage material. The cytosolic (eukaryotic) type ACCase provides malonyl-CoA for the synthesis of very long fatty acids ( 20 or more carbon atoms), flavonoids and anthocyanins. Traditionally, these compounds are classified as secondary metabolites but a detailed analysis of plant cytosolic ACCases has proved their essential role in plant development (Zuther et al., 2004).

Animal and fungal ACCases are exclusively of eukaryotic type with one putative exception, the nematode Turbatrix aceti enzyme, which has not been applied for biotechnological purposes so far (Meyer and Meyer, 1978; Barber et al., 2005). Both animals and fungi have two isoforms of ACCases designated as ACC1 and ACC2. The best studied ones are human enzymes: ACC1 (also named ACC-alpha, coded by $A C A C A$ gene) located in the cytosol, abundant in lipogenic tissues such as liver or adipose, and ACC2 (ACC-beta, coded by $A C A C B$ gene) associated with mitochondria and highly abundant in skeletal muscles and heart (Brownsey et al., 2006). The ACC2 


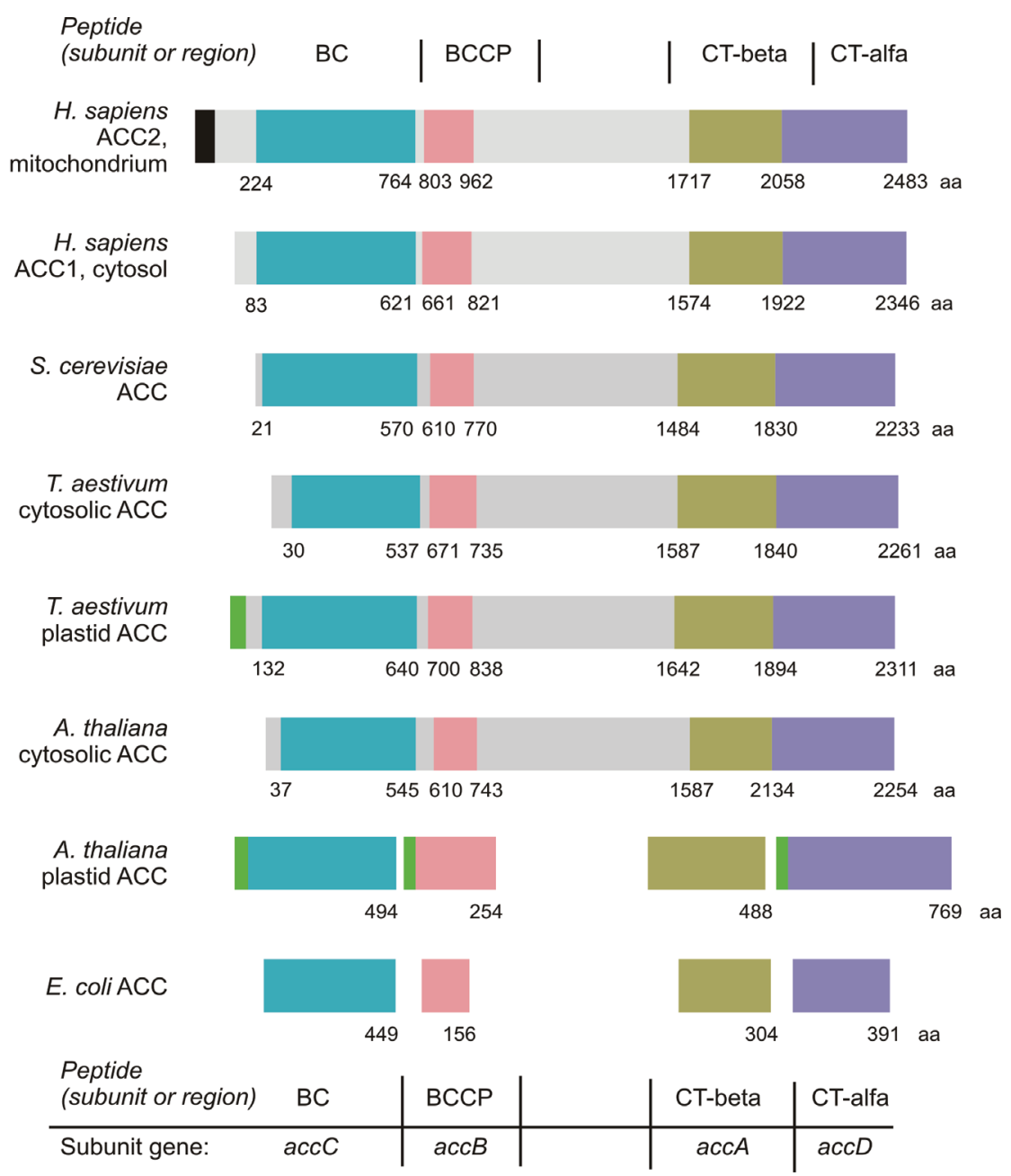

Fig. 3. Architecture of acetyl-coenzyme carboxylases. The organization of subunits and domains arrangement of ACCases from bacteria, dicotyledonous plants, monocotyledonous plants, yeast and humans are presented. Proteins synthesized in cytosol and targeted to organelles are presented in immature form, including targeting peptide marked with green for plastid targeting peptide, and with black for peptide responsible for directing protein to the outer mitochondrial membrane. Dicotyledonous acc $A$ is a plastid genome gene for CT-beta subunit whereas all other peptides are coded on nuclear genome (Tong 2005)

form has an N-terminal mitochondria targeting peptide which docks the enzyme on the outer mitochondrial membrane exposed to the cytosol (Abu-Elheiga et al., 2000). The two forms of ACCases play different roles in human cellular homeostasis, which results from differences in genes regulation, proteins activity and product allocation. The cytosolic ACC1 produces malonyl-CoA for FAS and, therefore, both enzyme activity and gene transcription are increased under anabolic conditions. The mitochondria-associated ACC2 is mainly involved in the regulation of fat beta-oxidation pathway by inhibiting carnitine palmitoyl transferase-1 (CPT-1) which controls the long chain fatty acyl-CoA transport into mitochondria. Hence, the two isoforms of human ACCases play opposite roles in cellular energy homeostasis.
A high diversity of bacterial metabolic pathways does not allow a brief, general characterization of ACCase and the impact of its product on cellular processes in this group of organisms. Escherichia coli, the model bacteria of well-studied biochemistry and molecular biology as well as a "working horse" of biotechnology, provides some introductory information on ACCase relations with other biochemical pathways. In E. coli, the ACCase substrate - acetyl-CoA is directed mainly to tri-carboxylic acids cycle, leaving only a small fraction for malonyl-CoA synthesis and, subsequently, for fatty acids production. Multiple regulatory mechanisms control the amount of acetyl-CoA entering FAS pathway, as well as ACCase gene expression and the enzyme activity. All these factors are tightly adjusted to cell growth rate, the demand 
for phospholipids and other macromolecules. One of the recently discovered mechanisms that coordinate carboxyl transferase genes expression with the rate of cell metabolism depends on the specific interactions between $\mathrm{CT}$ subunits and transcripts of their genes (Meades, Jr. et al. 2010).

$E$. coli model is not capable of covering the whole diversity of bacterial biochemistry and, in other bacterial species, the malonyl-CoA pool may be directed to other pathways and be spent exclusively on FAS. Whereas a global demand for renewable fuel makes fatty acids production in bacteria the most obvious goal of biotechnology, the other bacterial routes of malonyl-CoA processing may also find practical applications. One of such promising pathways is the 3-hydroxypropionate cycle in autotrophic carbon fixation present in some bacteria and archaeons, which allows them to fix inorganic carbon dioxide (or bicarbonate), as shown schematically in Figure 4 (Zarzycki et al., 2009; Tabita, 2009). The ability to synthesize all building blocks of life from the most oxidized form of carbon is of particularly great biotechnological value as a method for production of an unlimited variety of compounds or carbon dioxide sequestration. Till now, only a small part of bacterial metabolic pathways have been applied in biotechnology but fast development in this field will certainly attract attention to this type of uncommon biochemical pathways.

\section{ACCase in bacteria and algae-based biotech projects}

Acetyl-CoA carboxylase as the enzyme responsible for the first committed reaction in the fatty acid biosynthesis was also suggested to be the major rate-controlling step of this pathway. Therefore, the identification of ACCase encoding genes opened the door to engineering organisms to be used as bioreactors for fatty acid production. While this field of biotechnology is expanding, driven by the increasing needs for renewable sources of energy, malonyl-CoA, the direct product of the reaction catalyzed by ACCase, also serves as a universal precursor for a variety of other high-value compounds. They include divergent polyketides and flavonoids, both found at the center of interest owing to their various biochemical activities widely applied in pharmaceutical industry.

The first metabolic engineering application of ACCase was the overexpression of a set of four E. coli genes encoding four bacterial subunits of this enzyme
(Davis et al., 2000). All genes were assembled in an artificial operon under bacteriophage T7 promoter. Grouping all genes under the same tightly controlled, inducible promoter on a low copy plasmid was essential for the project as an abnormal ratio and high concentration of ACCase subunits are harmful to bacterial growth. The transgenic ACCase subunits were produced in near equimolar ratios, forming a mature, catalytically active complex in vivo. Their high abundance resulted in around 50times higher enzyme activity within the cells. Interestingly, 50-times increased amounts of transgenic BCCP were efficiently processed by native bacterial biotin protein ligase, an enzyme responsible for the posttranslational modification essential for the ACCase activity. In this project, the free fatty acids (FFAs) were excreted into the culture medium which became a high capacity metabolic sink storing the product and separating it from the engineered pathway and other pathways that are essential for cell homeostasis. This redirecting and uncoupling of FFAs from phospholipids synthesis was achieved through the overexpression of modified bacterial tes $A$ gene coding for thioesterase I (TesA) lacking the leader peptide that targets it to the cellular periplasm. The presence of bacterial TesA in the cytosol is the clue factor that redirects FAS to FFAs secretion to the culture medium (Cho and Cronan, Jr., 1995). TesA cleaved the long chain products of FAS pathway from acyl carrier protein (ACP), which allowed the export of FFAs into the medium. While reduced cellular acyl-ACP concentrations stimulated FAS by decreased feedback inhibition, high FFA concentration channeled the products into the extracellular metabolic sink. The whole system became a functional bioreactor for FFAs production. In this study, the 50-times increased activity of the enzyme resulted in near 100-times higher level of malonyl-CoA in the cell. In consequence, FAS was "pumped up" according to previous expectations, but even with the use of a high capacity sink, the rate of FFA synthesis increased only 6 times. This was probably due to some later steps of the pathway such as reactions catalyzed by enoyl reductase and 3-ketoacyl ACP synthase. Nevertheless, this pilot work shaped the path for ACCase metabolic engineering in bacteria and thus proved that this multisubunit enzyme might be reconstituted in bacteria and is able to efficiently produce fatty acids.

Some of the reoccurring problems were solved by equipping the $E$. coli strain overexpressing ACCase ge- 


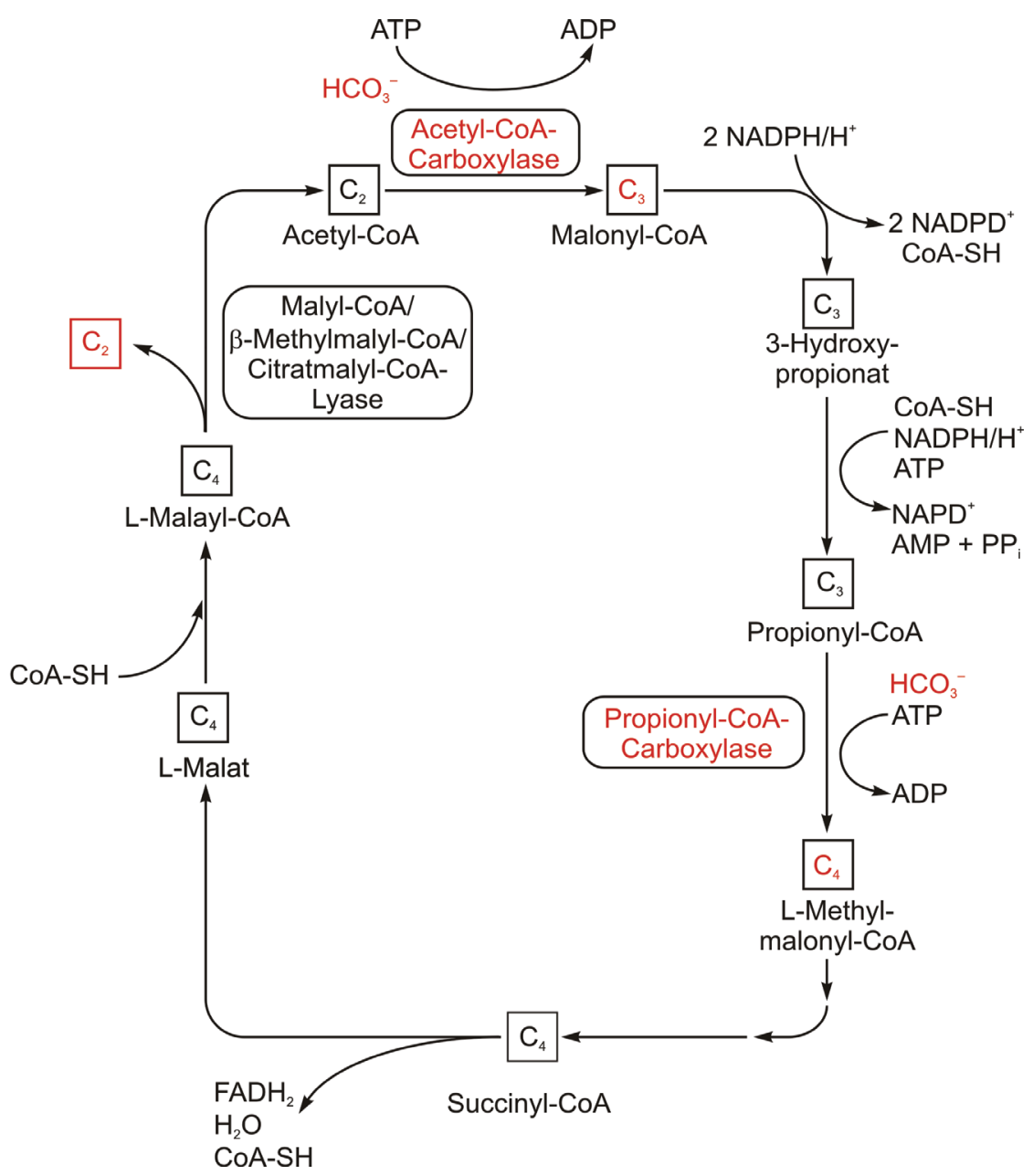

Fig. 4. A 3-hydroxypropoinate cycle from autotrophic carbon fixation (Zarzycki et al. 2009). Acetyl-coenzyme A and propionyl-coenzyme A carboxylases facilitate an inorganic carbon dioxide fixation forming glyoxylate

nes with additional modifications, targeted to directly increase fatty acids production efficiency (Lu et al., 2008). Firstly, $E$. coli gene fadD coding for acyl-CoA synthetase was knocked-out since the deficiency of its product resulted in the inhibition of the fatty acids degradation pathway. Secondly, overexpression of $E$. coli endogenous thiosterase gene was, as in a previous study (Davis et al., 2000), intended to partially block feedback inhibition caused by increased amounts of long chain fatty acylACP. Thirdly, the expression of plant thioesterase gene from Cinnamomum camphorum increased the abundance of short chain fatty acids, which is beneficial for biodiesel production. Such $E$. coli strain was capable of efficient production of fatty acids, achieving productivity of $0.024 \mathrm{~g}$ of fatty acids per $1 \mathrm{~h}$ and $1 \mathrm{~g}$ of dry mass. This result is within the order of magnitude of ethanol-specific productivity in commercial processes. A detailed analysis revealed that more than $50 \%$ of the overproduced fatty acids were accumulated in free form and, surprisingly, most of them were sequestered inside cells or in periplasm. The authors suggest that such a distribution was the result of re-absorption of fatty acids firstly excreted into the medium.

Recently, much effort has been put into improving the malonyl-CoA level in $E$. coli cells for the production of its other derivatives (Zha et al., 2009). The study involved coexpression of Corynebacterium glutamicum ACCase and $E$. coli acetyl-CoA synthetase (Acs) encoding genes oriented to increase intracellular malonylCoA level, combined with inactivation of competing, acetyl- and malonyl-CoA consuming pathways. Acs is an enzyme that produces acetyl-CoA as a result of acetate assimilation process within bacterial cells. Genes coding for phosphotransacetylase and acetate kinase i.e. the enzymes responsible for reversal conversion of acetyl-CoA to acetate were deleted from $E$. coli genome to improve 
the effect of Acs activity. Simultaneously, bifunctional alcohol/aldehyde hydrogenase gene was deleted, thus eliminating another way of acetyl-CoA utilization by ethanol synthesis. The modified bacterial strain showed 15 -fold higher malonyl-CoA concentration accompanied with decreased concentration of acetyl-CoA. Although in this case carbon flux was effectively redirected toward malonyl-CoA synthesis, its utility in large scale biosynthesis still awaits verification. The engineered strain was transformed with phloroglucinol synthase gene phlD, which enables conversion of three molecules of malonyl-CoA into phloroglucinol. Phloroglucinol is a polyketide used, for example, as an intermediate for pharmaceuticals production, as a smooth muscle relaxant or as an antioxidant. Unfortunately, the observed production efficiency of $1.28 \mathrm{~g} / \mathrm{L}$ (a 2.5 -fold change compared to control strain with phID expression) was much lower than expected, compared to malonyl-CoA concentration improvement, probably due to product toxicity to bacterial cells. Interestingly, a much more effective phloroglucinol production strategy has been recently shown using an $E$. coli strain which overexpressed marA (multiple antibiotic resistance) gene which increased bacterial tolerance to the product (Cao et al. 2011). In this study, coexpression of $p h I D$ and four ACCase subunits genes demonstrated the productivity of $3.8 \mathrm{~g} / \mathrm{L}$ (2.1-fold change to the control strain) at large scale fed-batch fermentation conditions. This was the highest phloroglucinol production efficiency reported to date and proved that single ACCase overexpression is sufficient to greatly improve the production rate of the entire synthesis pathway.

Similarly, coexpression of two genes of $C$. glutamicum ACCase proved to greatly increase the overall production efficiency of flavanones, the direct precursors of the vast majority of flavonoids (Miyahisa et al., 2005). In this study (2S)-naringenin and (2S)-pinocembrin were synthesized in $E$. coli by employing an artificial biosynthetic cluster of genes that included phenylalanine ammonia lyase from yeast, cinnamate/coumarate:CoA ligase from the actinomycete Streptomyces coelicolor, chalcone synthase from a licorice plant, and chalcone isomerase from a Pueraria plant. A similar effect, related to an increased availability of malonyl-CoA in the cell, was observed during the production of five different plant flavonones accompanied by expression of Photorhabdus $1 u$ minescens ACCase gene (Leonard et al., 2007). In this case, the increased ACCase activity present in $E$. coli cells was additionally supported by overexpression of bacterial gene coding for Acs. The achieved flavanone production levels were also claimed to be the highest from any recombinant system reported to date.

Nowadays, much focus is also put toward an efficient biosynthesis of fatty acids, since these compounds are highly in demand for biofuels production. Apart from $E$. coli, cyanobacteria that make use of solar energy and $\mathrm{CO}_{2}$ seem to be good hosts for this purpose. Cyanobacteria are the evolutionary ancestors of plant plastids, therefore FAS machineries of both are similar (Vothknecht and Westhoff, 2001). In a recent study, genetically modified cyanobacteria were used to produce and secrete fatty acids mimicking a process that occurs in plants, where FFAs are synthesized in plastids and subsequently exported to the cytosol (Liu et al., 2011). The strategy involved numerous deletions in Synechocystis sp. wild type strain. One goal was to prevent production of substantial by-products that would compete with production of FFAs. For this purpose, two poly-3hydroxybutyrate (PHB), two cyanophycin, acyl-ACP synthetase (Aas) and phosphotransacetylase (Pta) encoding genes were disabled. Other deletions of hemolysin-like surface layer protein gene and penicillin-binding protein (PBP2) gene (responsible for peptidoglycan layer assembly) aimed to enhance the secretion of FFAs into the medium. Simultaneously, some genes were introduced to increase FFA production, including a set of $E$. coli ACCase genes and E. coli thioesterase I gene. The TesA protein was, as in previous cases, modified by deletion of the periplasm-targeting peptide. Finally, to adjust the chain length of secreted FFAs for further fuel production, a set of additional plant thioesterases which hydrolyze acyl-ACP thioester bonds with preference to particular fatty acid attached was also introduced. The results show that FFA-secreting cyanobacteria provide a promising, yet still not fully developed technology enabling efficient production of this compound. Their attractiveness stems from the fact that synthesized FFAs may be directly collected from the culture medium, and that their chemical properties may be adjusted according to further needs. This complex metabolic engineering study again illustrates the significant role of ACCase in increasing fatty acids biosynthesis.

Other interesting candidates for biodiesel production are microalgae that can naturally accumulate substantial amounts of energy-rich compounds, like triacylglycerols 
(TAG) or starch. Although in some microalgal species, lipid content may reach up to $75 \%$ of their dry weight, it is usually conditioned by the occurrence of some environmental stress such as a lack of nitrogen. Simultaneously, the proliferation rate is markedly decreased, and affects the overall productivity of the system (Hu et al., 2008). In order to combine high lipid content with relative ease of cultivation and high production efficiency, metabolic engineering trials were conducted. ACCase of the diatom Cyclotella cryptica became the first microalgal metabolic engineering target (Dunahay et al., 1996). This project greatly facilitated the development of microalgal expression vectors and transformation protocols for future projects. However, the overexpression of ACCase gene in this study did not enhance lipid production significantly, which suggests that some other rate-limiting steps of the pathway occur and must be identified and improved. Several other metabolic engineering studies aiming to improve TAG production in algae did not result in markedly better outcomes (Sheehan et al., 1998).

In summary, bacterial systems with ACCase overexpression were shown to be suitable for efficient production of various malonyl-CoA derived compounds, including fatty acids, polyketides, and flavonoids. Although overproduction of ACCase alone increases the total malonyl-CoA pool in the cells, the productivity required for the industrial scale may be obtained only with additional modifications that disable native regulatory and competing processes. While among the well known and widely utilized advantages of $E$. coli is the fact that they grow on a variety of inexpensive carbon sources and that the genetics and biochemistry of the bacterium has been well researched, cyanobacteria that use exclusively $\mathrm{CO}_{2}$ and solar energy have been found to be a good alternative to serve as hosts in the production processes. Photosynthetic algae based projects also seem to be very promising alternative, as those organisms are the most efficient biological producers of oil on the planet. Yet, microalgal technologies are still under development, and probably more complex metabolic engineering approaches will be required to enhance their lipid biosynthesis efficiency.

\section{ACCase - essential enzyme for oil production in plants}

The goals of acetyl-CoA carboxylase manipulations in plants are dual: (i) to increase the efficiency of plant oil production and (ii) to better understand the mechanisms regulating FAS pathway on molecular and genetic levels.

The role of biotin carboxyl carrier protein in fatty acids deposition in seeds was characterized by transgenic Arabidopsis thaliana plants of up- and down-activated gene for BCCP2 - an isoform predominantly expressed in flowers and developing seeds (Thelen and Ohlrogge, 2002). Down-regulation of this gene did not reveal a significant phenotype apart from a reduced level of the protein and a decreased, by $9 \%$, content of fatty acids in seeds. A weak phenotype might be explained by unaffected expression of gene for $\mathrm{BCCP} 1$ which partially complemented the silenced homologue. More significant were the results of seed-specific BCCP2 overexpression, where a twofold increase of BCCP2 was accomplished with $23 \%$ lower fatty acids content in seeds. A detailed analysis of these transgenic plants revealed that $A$. thaliana native biotin protein ligase - contrary to $E$. coli - did not process abnormal amounts of BCCP subunits and left the majority of them not biotinylated. Such immature peptides competed with the biotinylated ones in the formation of ACCase complexes, which resulted in the suppression of the overall enzyme activity in plastids. These transgenic plants with suppressed plastid ACCase activity in seeds became a model to study the relations between FAS and other pathways (Chen et al., 2009). Transcriptomic and proteomic analyses of this model have revealed that $A$. thalina response to low-oil phenotype occurred at both gene and protein level. Induction of storage proteins was one of the major alternations and proved that oil and protein synthesis share carbon intermediates. The suppression of ACCase is an exclusive method to study in vivo function of this enzyme, the knock-out of which is lethal.

Nicotiana tabacum transformed with a gene for BC under constitutive promoter $35 \mathrm{~S} \mathrm{CaMV}$ proved that overexpression of a gene for other subunit is less deleterious for plant development (Shintani et al., 1997). Transgenic plants were capable of tolerating even five times higher expression of biotin carboxylase gene than wild type plants. This high amount of ectopically expressed biotin carboxylase neither influenced the leaf oil content nor the expression of genes for other subunits, and the majority of overexpressed $\mathrm{BC}$ peptides remained in an uncomplexed form. The differences between biotin carboxylase and BCCP phenotypes result, most probably, from posttranslational processing of $\mathrm{BCCP}$. Whereas 
non-biotinylated $\mathrm{BCCP}$ molecules are inactive, the whole pool of $\mathrm{BC}$, including transgene originated peptides, is composed of identical, functionally valid molecules.

Overexpression of $\mathrm{BCCP}$ and $\mathrm{BC}$ genes proved that coordinated expression of ACCase subunits observed in wild type plants did not include mechanisms capable of adjusting subunits expression to the most abundant ones (Ke et al., 2000). Whereas plants with suppressed plastid ACCase activity clearly showed that seed oil content depends on malonyl-CoA availability in this organelle, the approach depending on one of plastid ACCase subunits overexpression was not able to increase the malonyl-CoA production in plastids.

This goal was achieved with another method, i.e. targeting the eukaryotic type cytosolic ACCase to plastids. A chimeric gene comprising translational fusion between a transit peptide of a small unit of ribulose bisphosphate carboxylase and $A$. thaliana cytosolic ACCase was used to direct cytosolic enzyme to plastids. Brassica napus transformed with this transgene under napin promoter gave plants of 10 - to 20 -folds higher ACCase activity in seeds (Roesler et al., 1997). A thorough examination proved that the heterologous transit peptide was able to efficiently direct the extremely large, 2254 amino acids long ACCase protein into plastids and that the protein was biotinylated to the level comparable with native cytosolic ACCase. The high ACCase enzymatic activity observed in developing seeds ( 10 - to 20 -fold increase) was, probably, a result of transgene efficient expression and a reduction in protein turnover due to its atypical location. The transgenic plants were of 5\% higher average seed oil content than the wild type plants and their oil was enriched in oleic acid. The relative small increase in oil content in comparison with the highly increased ACCase activity could have resulted from the shortage of FAS enzymes or proteins involved in fatty acids deposition in seeds. Still, rapeseed with $5 \%$ higher oil content in seeds provides advantages for agriculture and industry.

A similar approach based on the eukaryotic type ACCase directed to plastids was used in studies on potato metabolism (Klaus et al. 2004). The plants were transformed with a construct comprising a gene for A. thaliana eukaryotic ACCase fused on $\mathrm{N}$ - termini with a Rubisco small subunit transit peptide under $35 \mathrm{~S} \mathrm{CaMV}$ promoter. This promoter ensured constitutive and efficient expression of the transgene, the product of which was biotinylated and which was subsequently transported into plastids. Plastids from potato tuber cells are hosts for two processes: sequestration of the photosynthesis-originated carbohydrates in the form of starch and synthesis of fatty acids. The transgenic potato made it possible to study the crosstalk between the two pathways and unraveled that overexpression of plastid targeted eukaryotic ACCase lead to a much greater increase in fatty acids production than the blocking of starch synthesis. The transgenic plants revealed of 10 -fold higher ACCase activity and 5-fold higher content of TAG. The latter are the most abundant form of storage lipids and the final product of fatty acids processing. These results proved that potato tubers are capable of synthesizing storage lipids and the efficiency of this process depends on ACCase activity in plastids. This activity may be enhanced with a cytosolic enzyme provided with small, heterologous transit peptide. Contrary to subunit $\mathrm{BCCP}$, the transgenic eukaryotic type acetyl-CoA carboxylase was efficiently biotinylated, even when its abundance was one or two orders of magnitude higher than in normal cells. The efficient biotinylation seems to be characteristic of a eukaryotic type ACCase targeted to plastids as it was observed in plants from distinct families - Brassicaceae and Solanaceae; however, the nature of differences between $\mathrm{BCCP}$ and the eukaryotic type ACCase biotinylation efficiency remains obscure.

An analysis of transgenic plants with modulated acetyl-coenzyme A carboxylase activity suggests that an efficient production of malonyl-coenzyme $\mathrm{A}$ is an indispensable, but not sufficient, prerequisite for high oil content in plant storage organs. The oil content as a quantitative trait is determined polygenically by genes from multiple loci, which include genes for enzymes processing malonyl-coenzyme A into lipids or TGA, packing them into oil bodies, as well as genes participating in the maturation of the storage organ. A coordinated expression of these genes depends on specific transcription factors which might become convenient tools in plant biotechnology. Studies on transcriptional control of genes contributing to oil content in Glycine max seeds resulted in the isolation of two such transcription factors - GmDof4 and GmDof11 (Wang et al., 2007). Their phenotypes studied with transgenic $A$. thaliana revealed an $11-22 \%$ higher content of fatty acids in seeds, an increased content of lipids in seeds, and a bigger seeds size and seed weight. One of these transcription factors, GmDof4 was shown 
to enhance expression of biotin carboxylase gene without affecting the expression of other ACCase subunits. It is not clear how the enhanced expression of biotin carboxylase gene stimulated fatty acids biosynthesis, and whether the differences in expression of plastid ACCase subunits genes resulted from the heterologous system applied for analysis - G. max transcription factors expressed in $A$. thaliana plant, but the results proved that specific transcription factors efficiently enhance lipid content in seeds.

The plants with overexpressed G. $\max$ Dof transcription factors proved that this indirect method of gene expression manipulation may be applied for increasing oil/lipid content in storage organs. The knowledge of transcriptional regulation of fatty acids synthesis in plants are not satisfactory and more detail studies on promoters of genes involved in FA synthesis and seed development are necessary to develop this technology. What seems especially promising, although not yet tested, is a combination of the two approaches - overexpression of a chimeric gene for plastid targeted cytosolic ACCase accomplished with enhanced expression of specific transcription factors

\section{Plant ACCases and susceptibility for herbicides}

Herbicides from three chemically dissimilar groups: aryloxyphenoxypropionates (AOPP, fops), cyclohexanediones (CHD, dims) and phenylpyrazolines are widely used in agriculture to control monocot weeds (Poaceae family). The specificity of these herbicides depends on differences in plastid ACCases architecture, since only the eukaryotic type enzyme, built of a single, large peptide with three domains, is susceptible to herbicide inhibition. Such eukaryotic type ACCases in plastids are characteristic of Poaceae family where the herbicides action blocks the whole FAS pathway, which consequently causes death of the plant. Some monocot crops, such as wheat, are resistant to herbicides, although their plastids ACCases are still sensitive due to the rapid metabolism of the inhibitors. The isolation of an eukaryotic type plastid ACCase genes from wheat and using them for complementation of yeast $A C C 1$ null mutation enabled the studying of plant genes in a yeast system (Podkowinski et al., 2003; Gornicki et al., 1997; Joachimiak et al., 1997). An analysis of a series of chimeric ACCases composed of fragments of plastid and cytosolic enzymes of different herbicide sensitivity, located the specific amino acids responsible for herbicide sensitivity within the conserved region of carboxyl transferase domain (Nikolskaya et al., 1999; Zagnitko et al., 2001). The comparison of natural, herbicide-resistant maize plastid ACCase mutants with a susceptible, "wild type" enzyme and an engineered mutant of an altered, specific single amino acid proved that the resistance to herbicide depends on leucine substituting isoleucine in a position equivalent to 1705 aa of yeast ACCase.

The natural maize mutant used in this study is not unique, and till now, such mutants have been reported for 35 weed species (www.weedscience.org) (Devine and Skula, 2000). The analysis of mutants revealed that plastid ACCases resistance to herbicides is associated with the alternations of five amino acids from CT domain (Delye et al., 2005; Nikolau et al., 2003). Since herbicide resistance originates from natural, spontaneous mutations and spreads relatively easily, there is an urgent need for new herbicides active against the resistant weeds, and also for new, faster methods of these compounds' development. This progress depends greatly on analysis of carboxyl transferase interactions with inhibitors, substrates, and product on molecular level. As long as plant ACCase or its CT domain have not been crystallized, their structural analysis and molecular modeling base on the crystal structure of yeast CT domain (Tong, 2005; Zhang et al., 2003). According to this model, the CT domain is composed of two: $\mathrm{N}$ - and C-subdomains, which correspond to prokaryotic CT-alpha and CT-beta subunits, respectively, and both have $\beta-\beta-\alpha$ superhelix fold. Plants CT domains are expected to form head-to-tail dimers, which are the active form of enzyme, capable of binding and processing the substrate - acetylCoA. The interface of the CT domains within dimer is composed of highly conserved amino acids, which also create an active site, and are involved in interactions with thiol group of CoA. Based on molecular modeling and structure of related enzyme - propionylo-coenzyme A carboxylase, a biotin substrate is expected to approach the CT active site from $\mathrm{C}$-subdomain of the other CT monomer (Diacovich et al., 2004).

The studies on CT domain interactions with herbicides are also the most advanced for the yeast enzyme. The crystal structure of yeast carboxyl transferase domain complexed with herbicides from the aryloxyphenoxypropionate group (fops) revealed that herbicide 
docks on yeast CT dimer interface, in pocket within the active site region but without overlapping with the substrate binding region (Zhang et al., 2004). This herbicide binding pocket is created as a result of haloxyfop interactions with CT dimer and is associated with a large conformational change of the active site region and its vicinity. The modified conformation of a CT domain in complex with a herbicide is incompatible with substrate binding, which, probably, is the main mechanism of inhibition. Yet, the interactions in yeast and monocots are not identical, as the yeast enzyme is much less sensitive to the inhibition with herbicides active against monocots. Moreover, the yeast model does not explain the molecular mechanisms of resistance as none of the five amino acids which confer herbicide resistance, is in direct contact with haloxyfop. Also, the inhibition mechanism in the yeast enzyme is not completely clear as single and double mutants are of similar sensitivity. The inhibition caused by dim herbicides is even less understood (Tong, 2005).

The model of CT domain interactions with herbicides from APP class has been recently refined with computational modeling methods applied for Alopecurus myosuroides cytosolic ACCase with mutation W374C (Zhu et al., 2011). The mutated ACCase, with tryptophan 2027 (position without parenthesis numbered according $A$. myosuroides cytosolic ACCase) substituted for cysteine, reveals different levels of resistance to various APP herbicides with very small structural differences. Computational modeling of a wild type and mutant CT domains proved that whereas amino acid at position 2027 did not interact directly with APP herbicides, substituting tryptophan for cysteine at this position caused conformational changes in residues at position 1999 (Trp346), 2030 (Phe377), and 1699 (Tyr161). These two latter amino acids participate in APP herbicides binding with pi stacking. The role of tryptophan at position 2027 (Trp374) depends on pi stacking with tryptophan at 1999 (Trp346) which, subsequently, induces specific conformation of phenylalanine at 2030 (Phe377). Eventually, this induced conformation of phenylalanine 2030 is favorable for herbicide binding. The models generated for four APP herbicides of different half maximal inhibitory concentration value $\left(\mathrm{IC}_{50}\right)$ identified six amino acids involved in pi stacking and $\mathrm{H}$-bond formation between herbicide and CT domain. One of these amino acids tryptophan at position 1999 (Trp346) - is specific for pi stacking exclusively with benzoxazol ring of fenoxaprop. This additional interaction is probably responsible for fenoxaprop superior $\mathrm{IC}_{50}$ value as the benzoxazol ring is not present in any other of the analyzed herbicides. The consistent correlation between the binding free energy calculated for the four analyzed APP herbicides complexed with CT domain and their $\mathrm{IC}_{50}$ value reinforce the models.

As long as there are no crystallography data, the computational modeling and simulation are the only methods available for elucidation of the monocots ACCase inhibition mechanism on atomic level. The results obtained form computational techniques supported by a functional analysis and experimental approaches allow to design novel ACCase inhibitors for agriculture.

\section{Human ACCase as target in diseases treatment}

Humans have two acetyl-coenzymes A carboxylases that play opposite roles in cell energy metabolism. The functions of those enzymes in cellular biochemistry and human physiology are complex and cannot be limited to simply providing malonyl-CoA for FA production. The defects in their activity or their genes expression are associated with various diseases such as obesity, insulin resistance and diabetes as well as heart disease and cancer (Tong and Harwood, 2006; Folmes and Lopaschuk, 2007). One of the proposed medical applications of the enzymes depends on their inhibition, which is associated with inhibition of fatty acids synthesis and stimulation of fatty acids oxidation, and which, in consequence, might reduce cardiovascular risk in metabolic syndrome. Compound CP-610431 bipiperidine derivative, and its analog of higher metabolic stability CP-640186, isolated by high-throughput screening, are such inhibitors active against both ACCase isoforms (Harwood, Jr., et al. 2003). Kinetic studies on ACCase CP-610431 complex and the crystal structure of yeast CT domain - CP-640186 complex have revealed that the binding site for these inhibitors is located on the carboxyl transferase domain (Zhang et al., 2004). The crystal structure of human ACC2 CT domain enabled identification of amino acids responsible for inhibitor binding and elucidated the role of extended $\mathrm{C}$-terminus specific for human (metazoan) enzyme in stabilization of CT dimer interface and inhibitor binding region (Madauss et al., 2009). A series of modified CP-640186 derivatives designed on the basis of these structural data led to the discovery of novel human ACCase inhibitor named 
26h (spirolactones bearing 2-ureidobenzothiophene) of improved activity, which discriminated between ACC1 and ACC2 enzymes (Yamashita et al., 2011).

CP-610431 and its derivatives are not the unique inhibitors of human ACCases. Others compounds of similar activity include natural polyphenol (resveratrol) (Gnoni and Paglialonga, 2009) or macrocyclic polyketide (soraphen), which interacts with biotin carboxylase domain of ACCase and interferes with ACCase phosphorylation (Cho et al., 2010; Jump et al., 2011). Progress in the improvement of the properties of inhibitors depends on the assays for screening, validation and characterization of those compounds. A recently reported system based on yeast $A C C 1$ gene-replacement strains is inexpensive and capable of high-throughput screening of chemical libraries (Marjanovic et al., 2010). Identification of an inhibitor, using this method, relies on the comparison of the yeast growth rate in the presence and absence of the analyzed compound, where the growth rate is evaluated simply by measuring the yeast culture absorption at 580-620 $\mathrm{nm}$. The yeast strain used in this method has an inactivated $A C C 1$ gene complemented with a gene for eukaryotic ACCase of interest residing on yeast shuttle vector. Both human genes ( $A C C 1$ and $A C C 2$ ) complemented yeast mutation. However, strains with human ACC2 enzyme grew more slowly, probably, due to a lower expression level of the transgene. Human enzymes with fused His-tag complemented the yeast ACC1 deficiency in a similar manner as the unmodified, full length proteins, but the low expression level made the yeast system unsuitable for recombinant protein isolation. Human ACCases were produced in baculovirus/insect Sf9 cell expression system to be later used in enzymatic assays for verification of selected candidate inhibitors.

This chemical compounds screening system proved highly reliable in pilot tests, where yeast strains complemented with well-characterized eukaryotic type ACCases were grown in the presence of known inhibitors. This standardization of protocol and availability of well-characterized controls allowed screening of three chemical libraries that together comprised more than $33,000 \mathrm{com}$ pounds. The screen resulted in 38 potential inhibitors of human acetyl-coenzyme A carboxylase 2. Two of these candidate inhibitors were analyzed in vitro with enzymatic assays. The analysis revealed that one of them does not discriminate between human ACCases isoforms, while the second compound was confirmed to be a highly specific inhibitor of human ACC2 enzyme, unaffecting ACC1. The analysis of the second compound in yeast replacement system with a gene for chimeric ACCase suggests that the binding site for this inhibitor is located outside CT domain.

The yeast gene replacement system which includes a set of yeast strains with well-characterized eukaryotic ACCases could be applied for various purposes such as isolation of inhibitors of other specificity, search for enzymes resistant against given inhibitor or studies on enzyme - inhibitor interactions. The role of inhibitors is not limited to the disease treatment only; they also facilitate studies on ACCases roles in physiology or regulatory processes as it was demonstrated in studies on hypothalamic regulation of food intake (Hu et al. 2003). Also, RNA interference is used for discriminating between the functions of particular ACCases isoforms (Savage et al., 2006). This technology was used for elucidation of ACC1 role in cancer (Brusselmans et al., 2005). The method that enables versatile studies on ACCase genes functions in living organims relies on transgenic mouse lines with knock-out genes. A systemic $A C C 2$ knock-out was reported to cause continuous fatty acids oxidation, reduced hepatic and body fat mass and an increase in energy expenditure, which was associated with reduced skeletal and cardiac muscle malonyl-coenzyme A and reduced susceptibility for diet-induced obesity and diabetes (Abu-Elheiga et al., 2001; Abu-Elheiga et al., 2003; Choi et al., 2007). Recently a new mouse line has been generated, which enabled conditional deletion of ACC2 gene from skeletal muscles - the predominant site of this gene expression. This conditional ACC2 deletion did not support any of the previously reported effects (Olson et al., 2010), which probaly was associated with differences in gene knock-out technologies: whereas "whole body knock-out" mice possessed deleted gene region coding for biotin binding motif resulting in production of incorrect transcript and inactive, truncated protein, the recently generated mouse line with conditional $A C C 2$ deletion has a completely inactivated gene which does not produce any protein. ACCases application in medicine and pharmacology still needs more detail studies to better understand the role of these enzymes in human physiology as well as etiology and development of diseases. In such basic studies, biotechnology seems to play an auxiliary function. The advantages of biotechnological methods such as 
high-throughput and standardization make them suitable for inhibitor discovery/development projects.

\section{ACCase as a target in infectious diseases}

The infectious diseases associated with apicoplexan parasites affect a large portion of human population as they include malaria caused by Palsmodium falciparum and a weakened immune system and encephalitis caused by Toxoplasma gondii, which is present in $30 \%$ of the population. The two apicoplexan protozoans possess FASassociated ACCase located in apicoplast, a non-photosynthetic plastid. The enzyme is of eukaryotic type and reveals sensitivity to herbicides from the aryloxyphenoxypropionate group (Zuther et al., 1999; Jelenska et al., 2001). The most effective herbicide from this group clodinafop, inhibits the growth of $T$. gondii in human fibroblasts by $70 \%$ and eliminates the parasite in 2-4 days. The analysis of $T$. gondii ACCase in yeast replacement line mapped the susceptibility for inhibitor to CT domain. The similarity of positions of amino acids responsible for apicoplexan and plant enzymes sensitivity for the herbicide suggests that the molecular mechanisms of the two processes are also similar (Jelenska et al., 2002). The low toxicity to mammalian cells makes the herbicides from aryloxyphenoxypropionate group an excellent starting point for developing new drugs.

The elucidation of FOP herbicides inhibitory mechanism against apicoplexan protozoans unraveled their being targeted against an enzyme of eukaryotic type and could not serve as a starting point to develop bacterial ACCase inhibitors. The other compounds pseudopeptide pyrrolidine dione antibiotics such as moiramide B were identified to interact and inhibit bacterial, multi-subunits ACCase. The mutations within both carboxyl transferase subunits lead to a resistance against pyrrolidine dione, which reinforces the identification of the antibiotic target (Freiberg et al., 2004). The inhibitor was active against $E$. coli enzyme at nanomolar concentrations and revealed good activity against Bacillus subtilis, Staphylococcus aureus and Streptococcus pneumoniae as well. This broad spectrum of the anti-bacterial activity resulting from conservancy of bacterial ACCases is one of the advantages of the antibiotics from this group. Moreover, the high selectivity of moiramide B for bacterial ACCases is associated with weak inhibition of the rat enzyme.

Last years brought a number of discoveries of new antibiotics targeted against bacterial ACCase. This pro- gress was possible owing to the application of a highthroughput method combined with computational modeling. In one of these approaches, the whole bacterial cell based screening of a large collection of pre-selected compounds identified several inhibitors targeted against biotin carboxylase (Miller et al., 2009). All these inhibitors belong to pyridopyrimidines - a new class of antibiotic and are selective for bacterial biotin carboxylase.

The progress in new antibiotic discoveries is associted with the introduction of such techniques as fragment-based drug discovery and virtual screening based on 3D shape and electrostatic property similarity searching (Mochalkin et al., 2009; Waldrop, 2009). Whereas virtual screening allows pre-selection of candidate molecules based on computational modeling, the advantage of fragment-based drug discovery depends on using, in initial screens, collections of low molecular weight compounds prepared to facilitate further chemical modification with fragment growing, merging or morphing.

The other group of bacterial ACCase inhibitors was isolated with screening mixture-based combinatorial library against bacterial biotin carboxylase with Automated Ligand Identification System (Cheng et al., 2009). The selected compounds, namely bezimidazoles of affinity to biotin carboxylase, were confirmed as low micromolar enzyme inhibitors, and, subsequently, optimized with computer-aided drug design combined with a synthetic approach. The compounds obtained in result of such multi-step selection/modification procedure are potent and selective inhibitors of prokaryotic biotin carboxylase. The activity of all these inhibitors relies on interference with ATP binding to the enzyme.

The studies on new antibiotics are a response to the expansion of antibiotic-resistant pathogenic bacteria which today seems to be a major health problem. Recent progress in this field that can be seen in the discoveries of bacterial $\mathrm{BC}$ inhibitors has been associated with the drug rational design, for which high quality crystal structure of bacterial $\mathrm{BC}$ is an essential prerequisite. High-throughput methods are also important for these discoveries, as well as the application of large and preselected collections of compounds for initial screening.

\section{Conclusions}

Acetyl-coenzyme A carboxylase is being used in various biotechnological projects that include, inter alia, isolation and design of new drugs, antibiotics and herbi- 
cides which address the main needs of the human population, i.e. improvement of health care and increased food production. Also projects that apply transgenic ACCase to metabolic engineering are within the scope of contemporary interest in sustainable technologies and environment friendly production of energy.

Most of acetyl-coenzyme A carboxylase applications depend on the structural data which enable rationale, computer-aided modeling, prediction of interesting interactions and designing compounds targeted against specific regions of the enzyme. Pre-selected libraries are also used in projects based on high-throughput screenings where such pre-selection is based on ACCase structural data and functional characterization. Such studies on ACCases function and structure are still needed, especially on the human enzymes. Also transcriptional regulation of ACCase genes, their promoters and transcription factors controlling their expression need better characterization for their application in biotechnological projects.

\section{References}

Abu-Elheiga L., Brinkley W.R., Zhong L., Chirala S.S., Woldegiorgis G., Wakil S.J. (2000) The subcellular localization of acetyl-CoA carboxylase 2. Proc. Natl. Acad. Sci. USA 97: 1444-1449.

Abu-Elheiga L., Matzuk M.M., Abo-Hashema K.A., Wakil S.J. (2001) Continuous fatty acid oxidation and reduced fat storage in mice lacking acetyl-CoA carboxylase 2. Science. 291: 2613-2616.

Abu-Elheiga L., Oh W., Kordari P., Wakil S.J. (2003) AcetylCoA carboxylase 2 mutant mice are protected against obesity and diabetes induced by high-fat/high-carbohydrate diets. Proc. Natl. Acad. Sci. USA 100: 10207-10212.

Barber M.C., Price N.T., Travers M.T. (2005) Structure and regulation of acetyl-CoA carboxylase genes of metazoa. Biochim. Biophys. Acta. 1733: 1-28.

Brownsey R.W., Boone A.N., Elliott J.E., Kulpa J.E., Lee W.M. (2006) Regulation of acetyl-CoA carboxylase. Biochem. Soc. Trans. 34: 223-227.

Brusselmans K., De S.E., Verhoeven G., Swinnen J.V. (2005) $R N A$ interference-mediated silencing of the acetyl-CoAcarboxylase-alpha gene induces growth inhibition and apoptosis of prostate cancer cells. Cancer Res. 65: 6719-6725.

Cao Y., Jiang X., Zhang R., Xian M. (2011) Improved phloroglucinol production by metabolically engineered Escherichia coli. Appl. Microbiol. Biotechnol. 91: 1545-1552.

Chen M., Mooney B.P., Hajduch M., Joshi T., Zhou M., Xu D., Thelen J.J. (2009) System analysis of an Arabidopsis mutant altered in de novo fatty acid synthesis reveals diverse changes in seed composition and metabolism. Plant Physiol. 150: 27-41.

Cheng C.C., Shipps G.W. Jr, Yang Z., Sun B., Kawahata N., Soucy K.A., Soriano A., Orth P., Xiao L., Mann P., Black T. (2009) Discovery and optimization of antibacterial AccC inhibitors. Bioorg. Med. Chem. Lett. 19: 6507-6514.

Cho H., Cronan J.E., Jr (1995) Defective export of a periplasmic enzyme disrupts regulation of fatty acid synthesis. Biol. Chem. 270: 4216-4219.

Cho Y.S., Lee J.I., Shin D., Kim H.T., Jung H.Y., Lee T.G., Kang L.W., Ahn Y.J., Cho H.S., Heo Y.S. (2010) Molecular mechanism for the regulation of human ACC2 through phosphorylation by AMPK. Biochem. Biophys. Res. Commun. 391: 187-192.

Choi C.S., Savage D.B., Abu-Elheiga L., Liu Z.X., Kim S., Kulkarni A., Distefano A., Hwang Y.J., Reznick R.M., Codella R., Zhang D., Cline G.W., Wakil S.J., Shulman G.I. (2007) Continuous fat oxidation in acetyl-CoA carboxylase 2 knockout mice increases total energy expenditure, reduces fat mass, and improves insulin sensitivity. Proc. Natl. Acad. Sci. USA 104: 16480-16485.

Davis M.S., Solbiati J., Cronan J.E. Jr (2000) Overproduction of acetyl-CoA carboxylase activity increases the rate of fatty acid biosynthesis in Escherichia coli. Biol. Chem. 275: 28593-28598.

Delye C., Zhang X.Q., Michel S., Matejicek A., Powles S.B. (2005) Molecular bases for sensitivity to acetyl-coenzyme A carboxylase inhibitors in black-grass. Plant Physiol. 137: 794-806.

Devine M.D., Shukla A. (2000) Altered target sites as a mechanism of herbicide resistance. Crop Prot. 19: 881-889.

Diacovich L., Mitchell D.L., Pham H., Gago G., Melgar M.M., Khosla C., Gramajo H., Tsai S.C. (2004) Crystal structure of the beta-subunit of acyl-CoA carboxylase: structure-based engineering of substrate specificity. Biochemistry 43: 14027-14036.

Dunahay T.G., Jarvis H.H., Dais S.S., Roessler P.G. (1996) Manipulation of microalgal lipid production using genetic engineering. Appl. Biochem. Biotechnol. 57-58: 223-231.

Folmes C.D., Lopaschuk G.D. (2007) Role of malonyl-CoA in heart disease and the hypothalamic control of obesity. Cardiovasc. Res. 73: 278-287.

Freiberg C., Brunner N.A., Schiffer G., Lampe T., Pohlmann J., Brands M., Raabe M., Habich D., Ziegelbauer K. (2004) Identification and characterization of the first class of potent bacterial acetyl-CoA carboxylase inhibitors with antibacterial activity. J. Biol. Chem. 279: 26066-26073.

Gnoni G.V., Paglialonga G. (2009) Resveratrol inhibits fatty acid and triacylglycerol synthesis in rat hepatocytes. Eur. J. Clin. Invest. 39: 211-218.

Gornicki P., Faris J., King I., Podkowinski J., Gill B., Haselkorn R. (1997) Plastid-localized acetyl-CoA carboxylase of bread wheat is encoded by a single gene on each of the three ancestral chromosome sets. Proc. Natl. Acad. Sci. USA 94: 14179-14184. 
Gornicki P., Podkowinski J., Scappino L.A., DiMaio J., Ward E., Haselkorn R. (1994) Wheat acetyl-coenzyme A carboxylase: cDNA and protein structure. Proc. Natl. Acad. Sci. USA 91: 6860-6864.

Harwood H.J., Jr, Petras S.F., Shelly L.D., Zaccaro L.M., Perry D.A., Makowski M.R., Hargrove D.M., Martin K.A., Tracey W.R., Chapman J.G., Magee W.P., Dalvie D.K., Soliman V.F., Martin W.H., Mularski C.J., Eisenbeis S.A. (2003) Isozyme-nonselective $N$-substituted bipiperidylcarboxamide acetyl-CoA carboxylase inhibitors reduce tissue malonyl-CoA concentrations, inhibit fatty acid synthesis, and increase fatty acid oxidation in cultured cells and in experimental animals. J. Biol. Chem. 278: 37099-37111.

Hu Q., Sommerfeld M., Jarvis E., Ghirardi M., Posewitz M., Seibert M., Darzins A. (2008) Microalgal triacylglycerols as feedstocks for biofuel production: perspectives and advances. Plant J. 54: 621-639.

Hu Z., Cha S.H., Chohnan S., Lane M.D. (2003) Hypothalamic malonyl-CoA as a mediator of feeding behavior. Proc. Natl. Acad. Sci. USA 100: 12624-12629.

Jelenska J., Crawford M.J., Harb O.S., Zuther E., Haselkorn R., Roos D.S., Gornicki P. (2001) Subcellular localization of acetyl-CoA carboxylase in the apicomplexan parasite Toxoplasma gondii. Proc. Natl. Acad. Sci. USA 98: 2723-2728.

Jelenska J., Sirikhachornkit A., Haselkorn R., Gornicki P. (2002) The carboxyltransferase activity of the apicoplast acetyl-CoA carboxylase of Toxoplasma gondii is the target of aryloxyphenoxypropionate inhibitors. J. Biol. Chem. 277: 23208-23215.

Joachimiak M., Tevzadze G., Podkowinski J., Haselkorn R., Gornicki P. (1997) Wheat cytosolic acetyl-CoA carboxylase complements an ACC1 null mutation in yeast. Proc. Natl. Acad. Sci. USA 94: 9990-9995.

Jump D.B., Torres-Gonzalez M., Olson L.K. (2011) Soraphen A, an inhibitor of acetyl $\mathrm{CoA}$ carboxylase activity, interferes with fatty acid elongation. Biochem. Pharmacol. 81: 649-660.

Ke J., Wen T.N., Nikolau B.J., Wurtele E.S. (2000) Coordinate regulation of the nuclear and plastidic genes coding for the subunits of the heteromeric acetyl-coenzyme A carboxylase. Plant Physiol. 122: 1057-1071.

Klaus D., Ohlrogge J.B., Neuhaus H.E., Dormann P. (2004) Increased fatty acid production in potato by engineering of acetyl-CoA carboxylase. Planta 219: 389-396.

Leonard E., Lim K.H., Saw P.N., Koffas M.A. (2007) Engineering central metabolic pathways for high-level flavonoid production in Escherichia coli. Appl. Environ. Microbiol. 73: 3877-3886.

Liu X., Sheng J., Curtiss R. III (2011) Fatty acid production in genetically modified cyanobacteria. Proc. Natl. Acad. Sci. USA 108: 6899-6904.

Lombard J., Moreira D. (2011) Early evolution of the biotindependent carboxylase family. BMC Evol. Biol. 11: 232.

Lu X., Vora H., Khosla C. (2008) Overproduction of free fatty acids in E. coli: implications for biodiesel production. Metab. Eng. 10: 333-339.

Madauss K.P., Burkhart W.A., Consler T.G., Cowan D.J., Gottschalk W.K., Miller A.B., Short S.A., Tran T.B., Wil- liams S.P. (2009) The human ACC2 CT-domain C-terminus is required for full functionality and has a novel twist. Acta Crystallogr. D Biol. Crystallogr. 65: 449-461.

Marjanovic J., Chalupska D., Patenode C., Coster A., Arnold E., Ye A., Anesi G., Lu Y., Okun I., Tkachenko S., Haselkorn R., Gornicki P. (2010a) Recombinant yeast screen for new inhibitors of human acetyl-CoA carboxylase 2 identifies potential drugs to treat obesity. Proc. Natl. Acad. Sci. USA 107: 9093-9098.

Meades G. Jr, Benson B.K., Grove A., Waldrop G.L. (2010) A tale of two functions: enzymatic activity and translational repression by carboxyltransferase. Nucl. Acids Res. 38: 1217-1227.

Meyer H., Meyer F. (1978) Acyl-coenzyme A carboxylase of the free-living nematode Turbatrix aceti. 2. Its catalytic properties and activation by monovalent cations. Biochemistry 17: 1828-1833.

Miller J.R., Dunham S., Mochalkin I., Banotai C., Bowman M., Buist S., Dunkle B., Hanna D., Harwood H.J., Huband M.D., Karnovsky A., Kuhn M., Limberakis C., Liu J.Y., Mehrens S., Mueller W.T., Narasimhan L., Ogden A., Ohren J., Prasad J.V., Shelly J.A., Skerlos L., Sulavik M., Thomas V.H., VanderRoest S., Wang L., Wang Z., Whitton A., Zhu T., Stover C.K. (2009) A class of selective antibacterials derived from a protein kinase inhibitor pharmacophore. Proc. Natl. Acad. Sci. USA 106: 1737-1742.

Miyahisa I., Kaneko M., Funa N., Kawasaki H., Kojima H., Ohnishi Y., Horinouchi S. (2005) Efficient production of (2S)flavanones by Escherichia coli containing an artificial biosynthetic gene cluster. Appl. Microbiol. Biotechnol. 68: 498-504.

Mochalkin I., Miller J.R., Narasimhan L., Thanabal V., Erdman P., Cox P.B., Prasad J.V., Lightle S., Huband M.D., Stover C.K. (2009) Discovery of antibacterial biotin carboxylase inhibitors by virtual screening and fragment-based approaches. ACS Chem. Biol. 4: 473-483.

Nikolau B.J., Ohlrogge J.B., Wurtele E.S. (2003) Plant biotin-containing carboxylases. Arch. Biochem. Biophys. 414: 211-222.

Nikolskaya T., Zagnitko O., Tevzadze G., Haselkorn R., Gornicki P. (1999) Herbicide sensitivity determinant of wheat plastid acetyl-CoA carboxylase is located in a 400-amino acid fragment of the carboxyltransferase domain. Proc. Natl. Acad. Sci. USA 96: 14647-14651.

Olson D.P., Pulinilkunnil T., Cline G.W., Shulman G.I., Lowell B.B. (2010a) Gene knockout of Acc2 has little effect on body weight, fat mass, or food intake. Proc. Natl. Acad. Sci. USA 107: 7598-7603.

Podkowinski J., Jelenska J., Sirikhachornkit A., Zuther E., Haselkorn R., Gornicki P. (2003) Expression of cytosolic and plastid acetyl-coenzyme $A$ carboxylase genes in young wheat plants. Plant Physiol. 131: 763-772.

Podkowinski J., Sroga G.E., Haselkorn R., Gornicki P. (1996) Structure of a gene encoding a cytosolic acetyl-CoA carboxylase of hexaploid wheat. Proc. Natl. Acad. Sci. USA 93: 1870-1874.

Rawsthorne S. (2002) Carbon flux and fatty acid synthesis in plants. Prog. Lipid Res. 41: 182-196. 
Roesler K., Shintani D., Savage L., Boddupalli S., Ohlrogge J. (1997) Targeting of the Arabidopsis homomeric acetyl-co enzyme $A$ carboxylase to plastids of rapeseeds. Plant. Physiol. 113: 75-81.

Sasaki Y., Nagano Y. (2004) Plant acetyl-CoA carboxylase: structure, biosynthesis, regulation, and gene manipulation for plant breeding. Biosci. Biotechnol. Biochem. 68: 1175-1184.

Savage D.B., Choi C.S., Samuel V.T., Liu Z.X., Zhang D., Wang A., Zhang X.M., Cline G.W., Yu X.X., Geisler J.G., Bhanot S., Monia B.P., Shulman G.I. (2006) Reversal of diet-induced hepatic steatosis and hepatic insulin resistance by antisense oligonucleotide inhibitors of acetyl-CoA carboxylases 1 and 2. J. Clin. Invest. 116: 817-824.

Sheehan J., Dunahay T., Benemann J., Roessler P. (1998) A look back at the U.S. Department of Energy's Aquatic Species Program - Biodiesel from algae. Nat. Renew. Ener. Lab., Colorado: 1-3.

Shintani D., Roesler K., Shorrosh B., Savage L., Ohlrogge J. (1997) Antisense expression and overexpression of biotin carboxylase in tobacco leaves. Plant Physiol. 114: 881-886.

Tabita F.R. (2009) The hydroxypropionate pathway of CO2 fixation: Fait accompli. Proc. Natl. Acad. Sci. USA 106: 21015-21016.

Thelen J.J., Ohlrogge J.B. (2002) Both antisense and sense expression of biotin carboxyl carrier protein isoform 2 inactivates the plastid acetyl-coenzyme $A$ carboxylase in Arabidopsis thaliana. Plant J. 32: 419-431.

Tong L. (2005) Acetyl-coenzyme A carboxylase: crucial metabolic enzyme and attractive target for drug discovery. Cell Mol. Life Sci. 62: 1784-1803.

Tong L., Harwood H.J. Jr. (2006) Acetyl-coenzyme A carboxylases: versatile targets for drug discovery. J. Cell Biochem. 99: 1476-88.

Vothknecht U.C., Westhoff P. (2001) Biogenesis and origin of thylakoid membranes. Biochim. Biophys. Acta 1541: 91-101.

Waldrop G.L. (2009) Smaller is better for antibiotic discovery. ACS Chem. Biol. 4: 397-399.

Wang H.W., Zhang B., Hao Y.J., Huang J., Tian A.G., Liao Y., Zhang J.S., Chen S.Y. (2007) The soybean Dof-type transcription factor genes, GmDof4 and GmDof11, enhance lipid content in the seeds of transgenic Arabidopsis plants. Plant J. 52: 716-729.
Yamashita T., Kamata M., Endo S., Yamamoto M., Kakegawa K., Watanabe H., Miwa K., Yamano T., Funata M., Sakamoto J., Tani A., Mol C.D., Zou H., Dougan D.R., Sang B., Snell G., Fukatsu K. (2011) Design, synthesis, and structure-activity relationships of spirolactones bearing 2-ureidobenzothiophene as acetyl-CoA carboxylases inhibitors. Bioorg. Med. Chem. Lett. 21: 6314-6318.

Zagnitko O., Jelenska J., Tevzadze G., Haselkorn R., Gornicki P. (2001) An isoleucine/leucine residue in the carboxyltransferase domain of acetyl-CoA carboxylase is critical for interaction with aryloxyphenoxypropionate and cyclohexanedione inhibitors. Proc. Natl. Acad. Sci. USA 98: 6617-6622.

Zarzycki J., Brecht V., Muller M., Fuchs G. (2009) Identifying the missing steps of the autotrophic 3-hydroxypropionate CO2 fixation cycle in Chloroflexus aurantiacus. Proc. Natl. Acad. Sci. USA 106: 21317-21322.

Zha W., Rubin-Pitel S.B., Shao Z., Zhao H. (2009) Improving cellular malonyl-CoA level in Escherichia coli via metabolic engineering. Metab. Eng. 11: 192-198.

Zhang H., Tweel B., Tong L. (2004) Molecular basis for the inhibition of the carboxyltransferase domain of acetylcoenzyme-A carboxylase by haloxyfop and diclofop. Proc. Natl. Acad. Sci. USA 101: 5910-5915.

Zhang H., Yang Z., Shen Y., Tong L. (2003) Crystal structure of the carboxyltransferase domain of acetyl-coenzyme $A$ carboxylase. Science 299: 2064-2067.

Zhu X.L., Yang W.C., Yu N.X., Yang S.G., Yang G.F. (2011) Computational simulations of structural role of the active-site W374C mutation of acetyl-coenzyme-A carboxylase: multidrug resistance mechanism. J. Mol. Model. 17: 495-503.

Zuther E., Huang S., Jelenska J., Eilenberg H., Arnold E.M., Su X., Sirikhachornkit A., Podkowinski J., Zilberstein A., Haselkorn R., Gornicki P. (2004) Complex nested promoters control tissue-specific expression of acetyl-CoA carboxylase genes in wheat. Proc. Natl. Acad. Sci. USA 101: 1403-1408.

Zuther E., Johnson J.J., Haselkorn R., McLeod R., Gornicki P. (1999) Growth of Toxoplasma gondii is inhibited by aryloxyphenoxypropionate herbicides targeting acetyl-CoA carboxylase. Proc. Natl. Acad. Sci. USA 96: 13387-13392. 